\title{
A load history dependent model for fatigue crack propagation in Inconel 718 under hold time conditions
}

Erik Lundström, Kjell Simonsson, David Gustafsson and T. Månsson

\section{Linköping University Post Print}

\section{Tweet}

N.B.: When citing this work, cite the original article.

Original Publication:

Erik Lundström, Kjell Simonsson, David Gustafsson and T. Månsson, A load history dependent model for fatigue crack propagation in Inconel 718 under hold time conditions, 2014, Engineering Fracture Mechanics, (118), 17-30.

http://dx.doi.org/

Copyright: Elsevier

http://www.elsevier.com/

Postprint available at: Linköping University Electronic Press

http://urn.kb.se/resolve?urn=urn:nbn:se:liu:diva-104809 


\title{
A load history dependent model for fatigue crack propagation in Inconel 718 under hold time conditions
}

\author{
E. Lundström ${ }^{\mathrm{a}, *}$, K. Simonsson ${ }^{\mathrm{a}}$, D. Gustafsson ${ }^{\mathrm{b}}$, T. Månsson ${ }^{\mathrm{c}}$ \\ ${ }^{a}$ Division of Solid Mechanics, Department of Management and Engineering, Linköping University, SE-58183 \\ Linköping, Sweden \\ ${ }^{b}$ Siemens Industrial Turbomachinery AB, SE-61283 Finspång, Sweden \\ ${ }^{c}$ GKN Aerospace Engine Systems, SE-46181 Trollhättan Sweden
}

\begin{abstract}
Modelling of high temperature fatigue crack growth in Inconel 718 under the interaction of fast cyclic loading and hold times at maximum load has been conducted. A model, based on the concept of a damaged zone in front of the crack tip has been applied for three different temperatures, 550, 600 and $650{ }^{\circ} \mathrm{C}$, with good agreement for both calibration and validation tests. A statistical evaluation of 22 tests in total was also conducted, which shows that the developed model gives a reasonable scatter factor at a probability of failure of $0.1 \%$.
\end{abstract}

Keywords: Fatigue crack propagation, Inconel 718, Hold time effects, Crack growth modelling, Statistics

\section{Introduction}

\section{Nomenclature}

Latin

$a$ crack length

$\dot{a}_{c}$ cyclic crack growth rate

$\Delta a_{c}$ cyclic crack length range

$a_{o}$ original crack length

$\dot{a}_{t}$ time dependent crack growth rate

$\frac{\mathrm{d} a}{\mathrm{~d} N}$ crack growth per load reversal

$A_{c}, B_{c}$ cyclic fitting parameters

\footnotetext{
${ }^{*}$ Corresponding author. Tel: +46(0)13282475

Email address: erik.lundstrom@liu.se (E. Lundström)
} 
$B_{t}$ time dependent fitting parameter

$C_{c}, n_{c}$ cyclic Paris constants

$C_{0 t}, n_{t}$ time dependent Paris constants

$D$ damaged zone length

$D_{0}$ initial damaged zone length

$D_{\max }$ maximum damaged zone length

$\dot{D}_{c}$ cyclic damaged zone rate

$\Delta D_{c}$ cyclic damage zone range

$\dot{D}_{t}$ time dependent damaged zone rate

$F(), f(), \bar{f}(), G(), g(), h()$ functions of

$k$ constant

$K_{\max }$ maximum stress intensity factor

$\Delta K$ stress intensity factor range

$\dot{m}$ damage mechanism growth rate

$N$ number of cycles

$t$ time

$X$ threshold value for time dep. crack growth

$Y$ threshold value for cyclic crack growth

$R_{p 0.2}$ yield limit at $0.2 \%$ strain

$R_{\sigma}$ load ratio

$S_{c}$ cyclic crack growth scale function

$S_{t}, C_{t}$ time dependent crack growth scale functions

\section{Greek}

$\sigma$ stress

$\dot{\sigma}$ stress rate

$\Delta \sigma$ stress range

\section{Abbreviations}

HT hold time

CI confidence interval

High temperature fatigue crack propagation under hold time conditions is an important issue in many areas; most notably the gas turbine industry. In the strive for increasing turbine efficiency, the need for more accurate life prediction models becomes more and more important as higher thermal efficiency requires increasing turbine temperature. With increasing temperature the materials involved will exhibit more severe operating conditions, which calls for reliable crack propagation models to handle these loading situations.

High temperature hold times are frequently seen in gas turbines, either at cruise in aero engines or at slow "ramp up" or continuous power output in land based turbines. These hold times can 
greatly increase the fatigue crack growth rate, and it has been shown that this behaviour is due to material damage near the crack tip, causing the crack to grow by intergranular fracture, see e.g. [1-3]. Between hold times different types of loading can occur, e.g. sequences of continuous cyclic loading. It has also previously been shown that not only the crack growth rate during the hold times but also the cyclic crack growth is affected by the material damage, cf. [4] for surface cracks. Thus, in order to understand and model the interaction between hold times at high temperature and cyclic loading conditions in e.g., real engine operation cycles, one has to consider the complete load history of a component.

The effect of sustained loading on the crack propagation behaviour has been extensively studied, cf. [1, 2, 5-14], which all show that hold times at high temperature can have a devastating effect on the crack growth rate. The modelling of hold time effects has also been studied by a number of different authors, often by use of additive models based on Paris law $[15,16]$ with a cyclic part, based on pure cyclic crack growth, and a time dependent part, based on pure time dependent crack growth, see e.g. [5, 6, 17-21]. However, this approach has been shown in e.g. [4] to be questionable from a physical point of view, see also [22]. When the crack growth during the hold time is separated from the crack growth occurring during the unloading and reloading, it is found that significant embrittlement of the grain boundaries must have occurred [3]. This aspect was further discussed in [4] and [23] where it was concluded that not only the crack growth during the hold time but also the crack growth during load reversal was affected by the hold time period. Thus, an incorporation of the actual damage mechanism in the fatigue crack growth description is motivated. For such works, see e.g. [24-27]; and more recently [28, 29]. For some reviews of the area, the reader is referred to, e.g., [30-32].

The effect of the mechanisms causing the hold time effect is seen in a volume of material around the crack tip, here referred to as the damaged zone, which gets a lowered resistance to fatigue crack propagation compared to the unaffected material, see e.g. [22, 33-39]. However, it is to be noted that the damaged zone is not a normal degradation damage parameter but instead a length measure of the degraded zone. Measurements of the length of the damaged zone by changing load cycle type are discussed in e.g. [4, 40], and it has been found that its length varies with temperature and hold time, but that it is usually tenths of millimetres in a stabilized state. Further, it has also been shown that the ratio between transgranular and intergranular fracture depends on both temperature and hold time length [7].

In the present paper, a model for the high temperature fatigue crack propagation behaviour of Inconel 718 will be presented. The proposed model, which is based on the concept of a damaged zone in front of the crack tip, represents a further development of the one presented by Gustafsson et al. [29], by incorporating an incubation time. Such incubation times are observed in the beginning of hold times and after load reversals, before the crack growth begins to accelerate, as shown in, e.g., $[33,38,41]$ and as will also be shown later in the present paper. Further, in this paper an efficient test for calibrating the model is presented, as well as new validation tests. Finally, the application of the model on a total of 22 different tests (including tests from [3, 4, 23, 42] and new calibration and validation tests in the present paper) at three different temperatures, (550, 600 and $650{ }^{\circ} \mathrm{C}$ respectively) are presented and discussed with respect to e.g. statistical issues.

\section{Material and experimental procedure}

\subsection{Material data}

The material used in this test series was Inconel 718, a wrought polycrystalline nickel based superalloy with a high content of $\mathrm{Fe}$ and $\mathrm{Cr}$. The material was delivered in the form of bars with a diameter of $25.4 \mathrm{~mm}$ and was solution annealed for $1 \mathrm{~h}$ at $945^{\circ} \mathrm{C}$, followed by ageing for $8 \mathrm{~h}$ at $718{ }^{\circ} \mathrm{C}$ and $8 \mathrm{~h}$ at $621^{\circ} \mathrm{C}$ according to the AMS 5663 standard. Two batches of the 
material with different production dates were used, both with a grain size of approximately 10 $\mu \mathrm{m}$, these will in the following be mentioned as batch $\mathrm{nr}$. 1 and batch nr. 2, respectively.

\subsection{Experimental crack growth procedure}

Crack growth experiments were conducted on Kb-type specimens, see Fig 1, with rectangular cross sections of $4.3 \times 10.2 \mathrm{~mm}$. An initial starter notch of nominal depth of $0.075 \mathrm{~mm}$ and a total width of $0.15 \mathrm{~mm}$ for batch $\mathrm{nr}$. 1 was generated using electro discharge machining (EDM). For batch nr. 2 a depth of $0.15 \mathrm{~mm}$ and a total width of $0.30 \mathrm{~mm}$ was used. Before the high temperature testing was carried out, the specimens were fatigue precracked at room temperature at $R_{\sigma}=0.05$, in order to obtain a sharp semicircular crack. The fatigue crack growth testing was then carried out under load control using an MTS servo hydraulic machine with a maximum load capacity of $160 \mathrm{kN}$, using an Instron 8800 control system and the software WaveMaker. The furnace used was an MTS 652.01 with a three zone temperature controller 409.81. The crack propagation was monitored by the direct current Potential Drop (PD) technique according to ASTM E647 [43] using a Matelect DCM-1, 2 channel pulsed DCPD system. Further, all tests were done in laboratory air and at a load ratio of $R_{\sigma}=0.05$, at a maximum stress of about 60 $\%$ of $R_{p 0.2}$ for each individual temperature, see Table 1, and all load reversals were based on a $0.5 \mathrm{~Hz}$ sine wave.

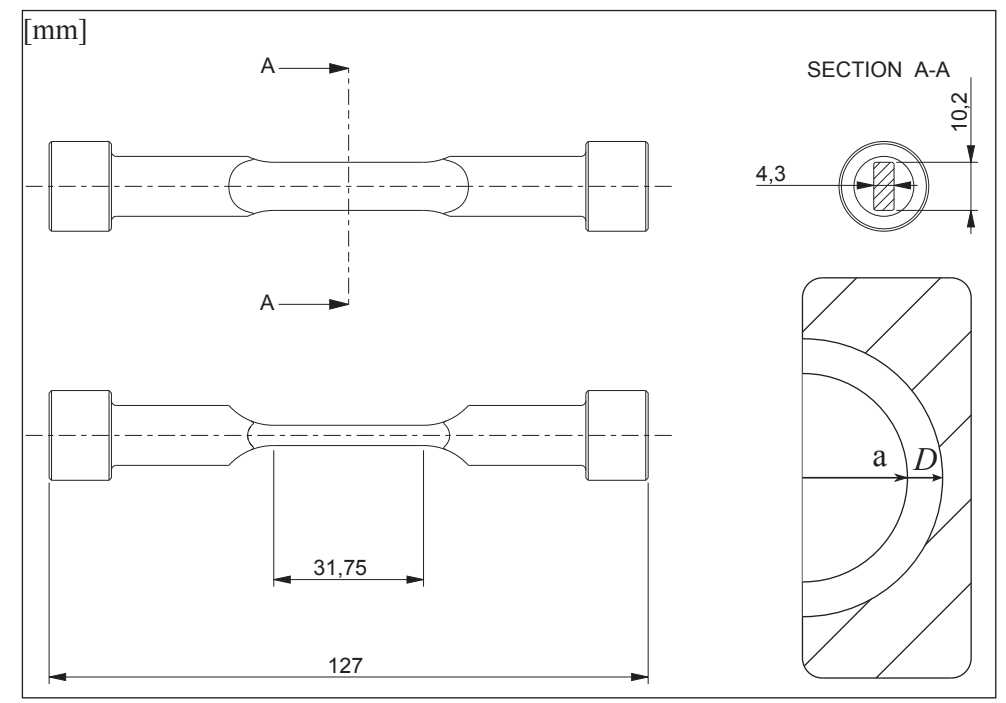

Figure 1: Drawing of Kb-specimen with the rectangular cross section marked with crack length and damaged zone.

Table 1: $R_{p 0.2}$ values for Inconel 718 for the temperatures used.

\begin{tabular}{ll}
\hline Temp. $\left[{ }^{\circ} \mathrm{C}\right]$ & $R_{p 0.2}[\mathrm{MPa}]$ \\
\hline 550 & 1000 \\
600 & 990 \\
650 & 960 \\
\hline
\end{tabular}

The measured PD ratio was translated to crack length, assuming a semi-circular crack front, which was confirmed post-mortem, through an experimentally obtained calibration function based on initial and final crack lengths measured on the fracture surface as well as by measured induced beach marks. This calibration function was then used on all other test specimens, and was confirmed by fracture surface measurement for some cases. It can be noted, that this 
method for monitoring the crack length has an accuracy of $0.01 \mathrm{~mm}$ crack length. Finally, an analytical stress intensity factor solution from ASTM E740 [44] was used. For a detailed record of the material and experimental conditions we also refer to $[3,4,23]$.

\section{Fatigue crack growth modelling}

As discussed in Section 1, the foundation of the time dependent crack growth behaviour is the lowering of the resistance against intergranular fatigue crack propagation through some mechanism affecting the grain boundaries. The affected volume of damaged material, i.e. the damaged zone, is used as the main modelling parameter to describe the increased crack propagation rates seen in the experiments. The length of the damaged zone depends on the history of hold times and interactions from load reversals. With these observations in mind, a history dependent phenomenological crack propagation model is proposed, with the damaged zone concept used for describing the observations discussed in Section 1. The damaged zone is in the modelling work defined as a local distance in front of the crack tip (registered by potential drop), see also Fig. 1, where the damaged zone and crack length are shown on the cross section of the test specimen.

Thus, qualitatively, with $a$ representing the crack length and $D$ the size of the damaged zone

$$
\begin{aligned}
a & =a_{0}+\int_{t_{0}}^{t} \dot{a} \mathrm{~d} t \\
\dot{a} & =F(a, D, \sigma, \ldots) \\
D & =D_{0}+\int_{t_{0}}^{t} \dot{D} \mathrm{~d} t \\
\dot{D} & =G(a, D, \sigma, \ldots)
\end{aligned}
$$

where $F$ and $G$ are functions to be defined. As seen in Eq. (1a) and (1c) the integration limits are the same for both $a$ and $D$, i.e. during a time step crack length and damaged zone are mutually affected by each other. Further, by restricting the attention to high frequency load reversals and constant load hold times, the crack growth and the damaged zone evolution are given by

$$
\begin{aligned}
& \dot{a}= \begin{cases}\dot{a}_{t} & \text { if }|\dot{\sigma}|<X \\
\dot{a}_{c} & \text { if }|\dot{\sigma}|>Y\end{cases} \\
& \dot{D}= \begin{cases}\dot{D}_{t} & \text { if }|\dot{\sigma}|<X \\
\dot{D}_{c} & \text { if }|\dot{\sigma}|>Y\end{cases}
\end{aligned}
$$

where the labelling "t" and "c" refer to time- and cyclic dependent quantities, respectively, and where $X$ and $Y$ are threshold values for when to apply time dependent or cyclic growth behaviour, depending on how "fast" the load is applied. In Eq. (2) and (3), the split of $\dot{a}$ and $\dot{D}$ into two parts is to be used when considering loading consisting of time dependent and cyclic parts. Each part is then used for the associated loading type, i.e. $a$ and $D$ are governed by one of the two options depending on loading type. However, as mentioned above, only the two extremities are examined in this this paper and therefore no values for $X$ and $Y$ are determined, for more details about such threshold values cf. [7, 9]. In detail the following relations are given 
for the time dependent part $(|\dot{\sigma}|=0)$

$$
\begin{aligned}
\dot{a}_{t} & =S_{t} \cdot C_{t} K_{\max }^{n_{t}} \\
S_{t} & =\frac{D}{D_{\max }} \\
C_{t} & =C_{0 t}\left(1-\exp \left(\frac{-B_{t}}{1-\frac{D}{D_{\max }}}\right)\right) \quad B_{t} \geq 0 \\
\dot{D}_{t} & =\dot{m}-\dot{a}_{t} \\
\dot{m} & =C_{t} K_{\max }^{n_{t}}
\end{aligned}
$$

where $D_{\max }$ represents the maximum size of the damaged zone under stationary conditions, and where $C_{0 t}, B_{t}$ and $n_{t}$ are fitting parameters. As can be seen, the fatigue crack propagation $\dot{a}$ is accompanied by a growth of the damaged zone $\dot{D}$, which is given by an additive combination of a growth term (due to oxidation) and a retardation term (due to crack propagation).

The function $C_{t}$ in Eq. (4c) describes the damaged zone in all aspects of its evolution, from undamaged material to fully intergranular growth. As indicated from mechanical testing, it takes some time for the crack growth to pick up speed, i.e. an incubation time can be observed at the beginning of pure time dependent crack growth tests and after load reversals, see Fig. 2 where a test with a mix of different hold times together with load reversals is seen, which cannot be considered as a regular $K$ threshold value effect, since no matter on what crack length one starts there is always seen a retarded crack growth rate for some time, cf. [33, 38, 41]. For more examples of this, cf. [4, 29]. The proposed $C_{t}$ expression takes its form by using the stabilised value at fully intergranular fracture, $C_{0 t}$ when $D=D_{\max }$, and by scaling it with a function of the amount of damaged zone present. The expression will thereby be at a low value at the build up of the damaged zone, i.e. in the beginning of the incubation time, and slowly reach larger values as the damaged zone evolves, finally stabilising at the value 1 when $D=D_{\max }$. If the damaged zone is reduced, e.g. by cyclic loading, the value of $C_{t}$ will decrease fast, decreasing the crack growth rate due to the exponential function. As seen in Eq. (4c), only 1 fitting parameter, $B_{t}$, is needed for acquiring an accurate description of the phenomenon, cf. the results presented in the next section.

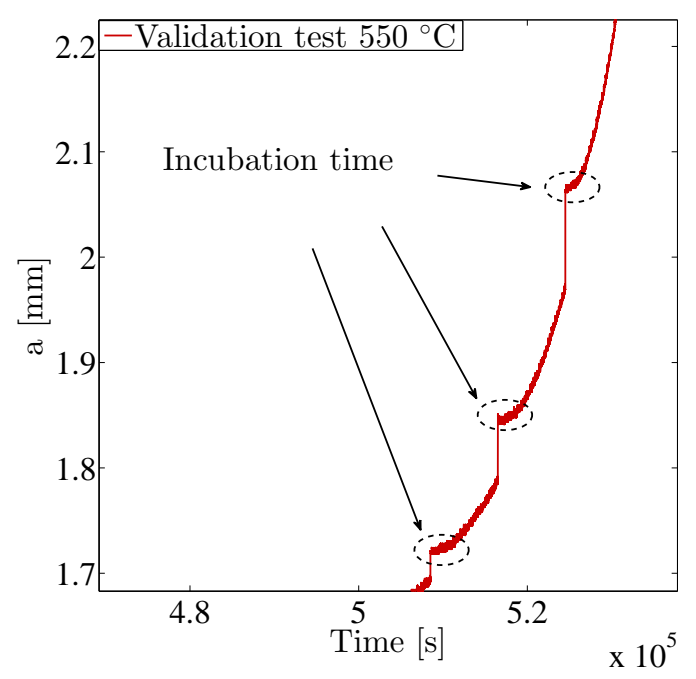

Figure 2: Three areas with incubation time after load reversals, from a zoom in on Fig. 9a.

The function $S_{t}$ controls to what extent the damaged zone affects the crack growth during the time dependent part, and should be able to describe the transient from pure cyclic loading, to, 
e.g., a block of hold times. With this choice of evolution equation for $D$, in combination with the monotonically increasing scaling function $S_{t}$, the evolution of $D$ will be stable in the sense that $D$ will never be larger than $D_{\max }$; for a discussion of the value of $D_{\max }$ see [23] and the next section.

For the cyclic part, a classical crack length description in combination with an associated damage zone reduction is proposed. In detail

$$
\begin{aligned}
\Delta a_{c} & =\int_{t_{1}}^{t_{2}} \dot{a}_{c} \mathrm{~d} t=\int_{0}^{1} \frac{\mathrm{d} a_{c}}{\mathrm{~d} N} \mathrm{~d} N \\
\Delta D_{c} & =\int_{t_{1}}^{t_{2}} \dot{D}_{c} \mathrm{~d} t=\int_{0}^{1} \frac{\mathrm{d} D_{c}}{\mathrm{~d} N} \mathrm{~d} N \\
\frac{\mathrm{d} a_{c}}{\mathrm{~d} N} & =S_{c} \cdot C_{c} \Delta K^{n_{c}} \\
S_{c} & =1+A_{c}\left(\frac{D}{D_{\max }}\right)^{B_{c}} \quad A_{c}, B_{c} \geq 0 \\
\frac{\mathrm{d} D_{c}}{\mathrm{~d} N} & =-\frac{\mathrm{d} a_{c}}{\mathrm{~d} N}
\end{aligned}
$$

where $C_{c}, n_{c}, A_{c}$ and $B_{c}$ are fitting parameters. As can be seen, the damaged zone will be progressively destroyed during repeated high frequency load reversals.

The function $S_{c}$ controls to what extent the damaged zone influences the cyclic part, and should e.g. be able to describe the transient from a hold time block to a block of pure cyclic loading. Since $S_{c}$ is to be a monotonically increasing function of $D$, and since it is to take the value 1 for the case of an "undamaged" crack-tip material, it may be given the form shown in Eq. (5d).

With the chosen evolution laws for $S_{c}$ and $S_{t}$, all crack growth rates will be at or between the values for pure transgranular and pure intergranular crack growth, respectively. As one can see from the model expression in Eq. (4b), $S_{t}$ will only reach its maximum value of 1 for sufficiently long hold times, which corresponds to pure time dependent crack growth, i.e. $D=D_{\max }$. The opposite applies for $S_{c}$ in Eq. (5d), starting at a high value depending on how close $D$ is to $D_{\max }$, and slowly decreasing towards its minimum value of 1 as the damaged zone is completely "consumed" by the damage zone evolution given by Eq. (5e). Thus, the model is robust in the sense that it will only predict crack growth within two well defined and classically wellknown rates, see Fig. 3. For a more comprehensive explanation of the different equations in the modelling work, we refer to [29]. 


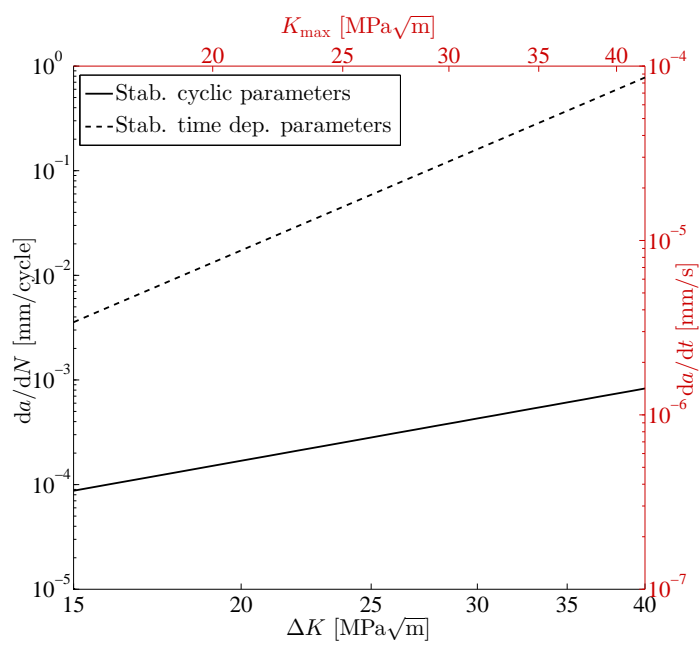

Figure 3: Limits of the model prediction for $550{ }^{\circ} \mathrm{C}$, indicated by the stabilised values from Table 2 .

As seen in Eq. (1), the behaviour is not modelled in a classical additive way. This is motivated by the history dependence observed in the material since, as previously discussed in e.g. [4] and [22], the crack growth is affected by the order/way in which the loading is applied. However, for the special case of a "high frequency" load spectrum without hold times (but with an existing damage zone), regular cycle counting techniques can be applied.

To see this, we note the following rewrite of Eq. (5b) and (5e)

$$
\begin{aligned}
\int_{D_{0}}^{D} \mathrm{~d} D_{c} & =\int_{a_{0}}^{a}-\mathrm{d} a_{c} \\
\Longrightarrow D & =D_{0}-\left(a-a_{0}\right)=\underbrace{D_{0}+a_{0}}_{k}-a
\end{aligned}
$$

and with this in Eq. (5d)

$$
\begin{aligned}
S_{c} & =1+A_{c}\left(\frac{D}{D_{\max }}\right)^{B_{c}} \\
& =1+A_{c}\left(\frac{k-a}{D_{\max }}\right)^{B_{c}}=h(a)
\end{aligned}
$$

Now, by incorporating Eq. (7) in Eq. (5c),

$$
\begin{aligned}
\frac{\mathrm{d} a_{c}}{\mathrm{~d} N} & =S_{c} \cdot C_{c} \Delta K^{n_{c}} \\
& =h(a) \cdot C_{c} \Delta K^{n_{c}} \\
& =h(a) \cdot C_{c}(\Delta \sigma \sqrt{\pi a} \cdot f(a, \ldots))^{n_{c}} \\
& =C_{c}\left(\Delta \sigma \sqrt{\pi a} \cdot f(a, \ldots) \cdot h(a)^{\left(1 / n_{c}\right)}\right)^{n_{c}} \\
& =C_{c}(\Delta \sigma \sqrt{\pi a} \cdot \bar{f}(a, \ldots))^{n_{c}}
\end{aligned}
$$

where $f(a, \ldots)$ is the ordinary geometry factor and where $\bar{f}(a, \ldots)$ plays the role of a new equivalent geometry factor $\left(f(a, \ldots) \cdot h(a)^{\left(1 / n_{c}\right)}\right)$. Thus, the evolution of crack length for cyclic load spectrums may be recast in an ordinary Paris law like form, which implies that regular cycle 
counting can be applied. Finally, it is to be noted that we have only modelled the condition where the depth and surface length relation are equal, i.e. a semi-circular crack front, $a / c=1$, and that the stress intensity factor considered is at the deepest point of the crack geometry.

\section{Results}

In this section, calibration and validation of the model is presented. The model has been implemented both in MATLAB [45] using the built in function ode45 (Dormand-Prince), and in FORTRAN, using the Runge-Kutta method, to solve the crack propagation equations. The optimisation of the model parameters was done by the commercial software LS-OPT [46], in combination with FORTRAN by minimisation of a least square based error function.

\subsection{Calibration}

Since it is of importance (especially in an industrial context) to be able to calibrate a model in an efficient way, a single test procedure has been developed (multiple tests to be used if more statistics are required).

During the calibration procedure, Fig. 4, different parts of the test are used to calibrate different parameters. This is done by first letting the test specimen be subjected to a block of continuous cycling to a crack length of $0.55 \mathrm{~mm}$, after which it is subjected to a constant loading till the specimen shows a linear behaviour in a log-log plot, which in our tests usually happens at a crack length of, say, $1.8 \mathrm{~mm}$. Finally it is subjected to a block of continuous cycling to a final crack length of about $2.6 \mathrm{~mm}$. From the transient found in the second cyclic block, the stabilised damaged zone $\left(D_{\max }\right)$ will be found, see [4]. The rest of the parameters can then be obtained from well specified parts of the test, i.e. $B_{t}$ from the transient at the beginning of the time dependent part, $C_{0 t}$ and $n_{t}$ from the stabilised part of the time dependent test, $A_{c}$ and $B_{c}$ from the transient during the start of the continuous cycling and finally $C_{c}$ and $n_{c}$ from the first continuous cycling and the stabilised level at the final continuous cycling, for which the damaged zone has been completely consumed. See Fig. 4 .

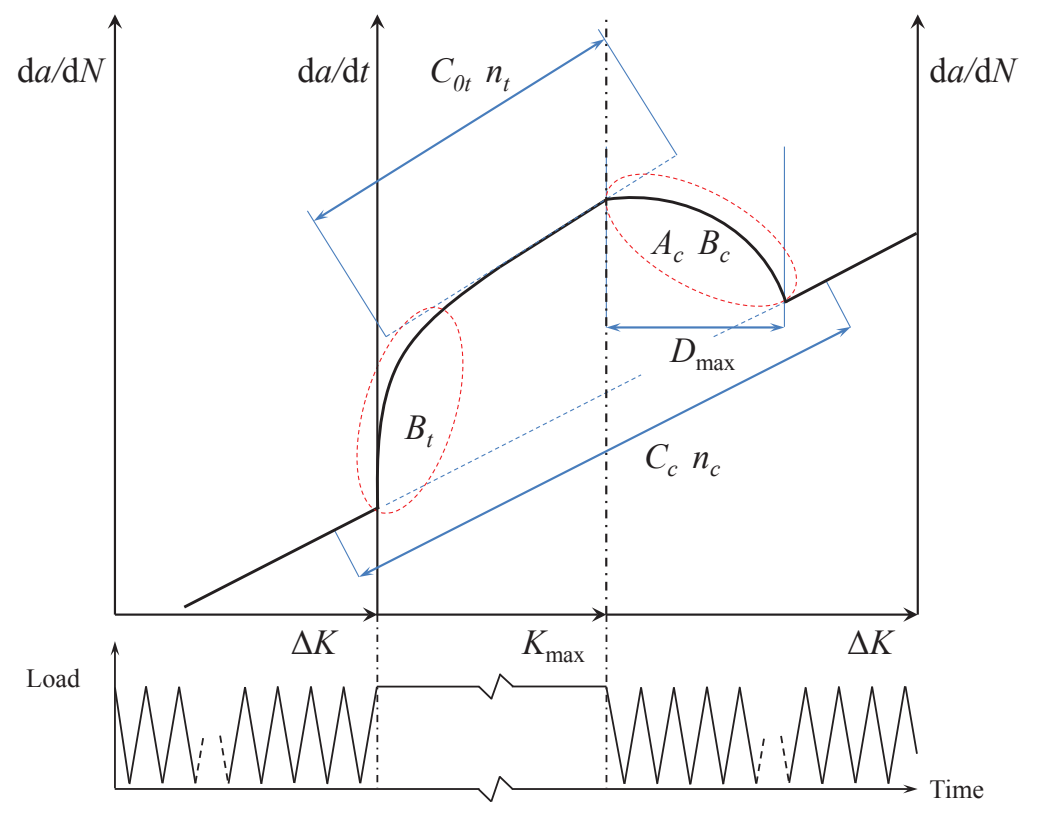

Figure 4: Calibration test and principle for parameter determination. 
It is to be observed that the same crack length intervals were used for all temperatures under study, but at different $K$ values due to different max load (60\% of $R_{p 0.2}$ ). A summary of all material parameters is shown in Table 2 below; please note that the stress intensity factors in Paris law must be given in $\mathrm{MPa} \sqrt{\mathrm{m}}$, and that the crack growth rates are in $\mathrm{m} / \mathrm{s}$ or $\mathrm{m} /$ cycle.

Table 2: Model parameters.

\begin{tabular}{llllllllll}
\hline Temp. $\left[{ }^{\circ} \mathrm{C}\right]$ & $\sigma_{\max }[\mathrm{MPa}]$ & $C_{0 t}$ & $n_{t}$ & $C_{c}$ & $n_{c}$ & $B_{t}$ & $A_{c}$ & $B_{c}$ & $D_{\max }[\mathrm{mm}]$ \\
\hline $550^{\mathrm{a}}$ & 650 & $3.9 \times 10^{-13}$ & 3.3 & $1.8 \times 10^{-10}$ & 2.3 & 0.35 & 303 & 2.7 & 0.5 \\
$550^{\mathrm{b}}$ & 650 & $3.9 \times 10^{-13}$ & 3.3 & $1.8 \times 10^{-10}$ & 2.3 & 0.71 & 282 & 3.1 & 0.5 \\
$600^{\mathrm{b}}$ & 600 & $9.1 \times 10^{-12}$ & 3.0 & $1.5 \times 10^{-10}$ & 2.5 & 0.42 & 283 & 3.0 & 0.7 \\
$650^{\mathrm{b}}$ & 550 & $6.0 \times 10^{-12}$ & 3.7 & $1.2 \times 10^{-11}$ & 3.5 & 0.51 & 180 & 3.0 & 0.8 \\
\hline
\end{tabular}

The results of the calibrations are seen in Fig. 5 for $550{ }^{\circ} \mathrm{C}$, in Fig. 6 for $600{ }^{\circ} \mathrm{C}$, and in Fig. 7 for $650{ }^{\circ} \mathrm{C}$. Please note that for the $550{ }^{\circ} \mathrm{C}$ a calibration test was only done for batch nr. 2, due to a shortage of test specimens, and therefore the calibration for batch $\mathrm{nr} .1$ at $550{ }^{\circ} \mathrm{C}$ was done using, (1) a baseline test (i.e. pure cyclic loading), (2) a pure time dependent crack growth test, and finally (3) a "down hill transient" from a block test with $2160 \mathrm{~s}$ hold time; for more information about this calibration procedure and the tests used we refer to [4, 29] and Section 4.2 .

\footnotetext{
abatch nr. 2

b batch nr. 1
} 


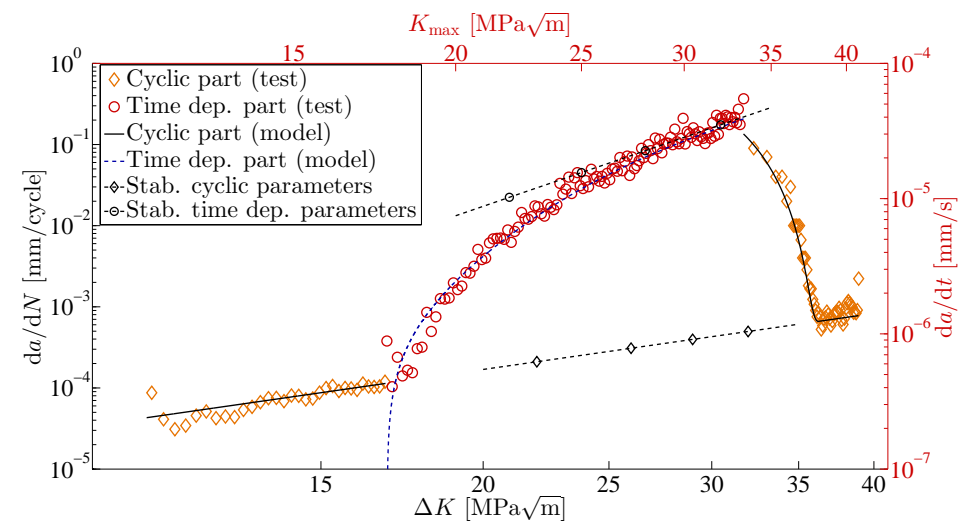

(a)

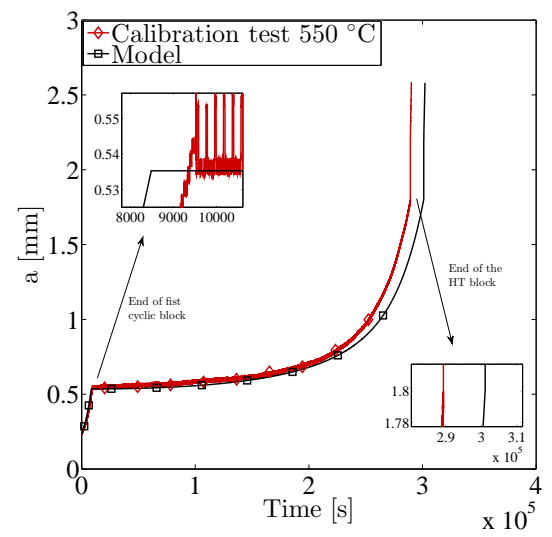

(b)

Figure 5: Calibration test for $550{ }^{\circ} \mathrm{C}$. (a) Calibration of parameters and (b) crack length vs. time. 


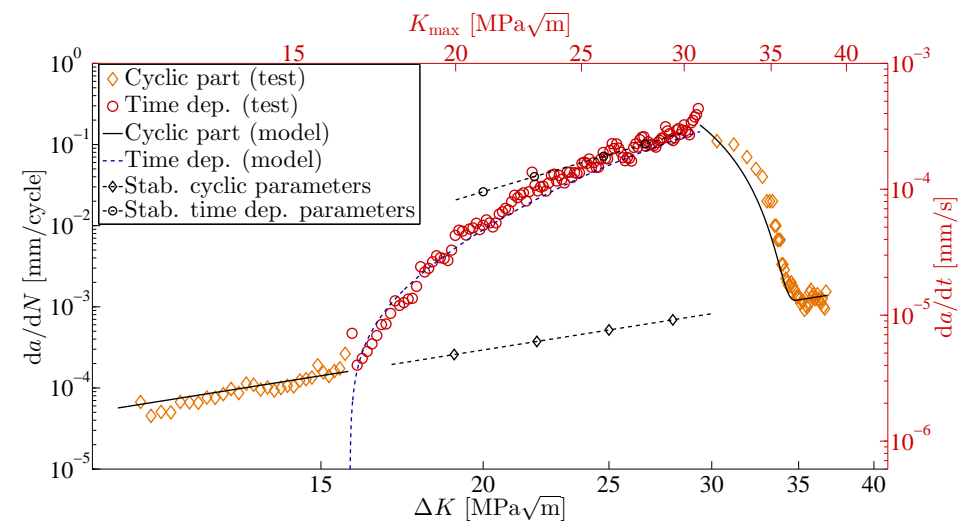

(a)

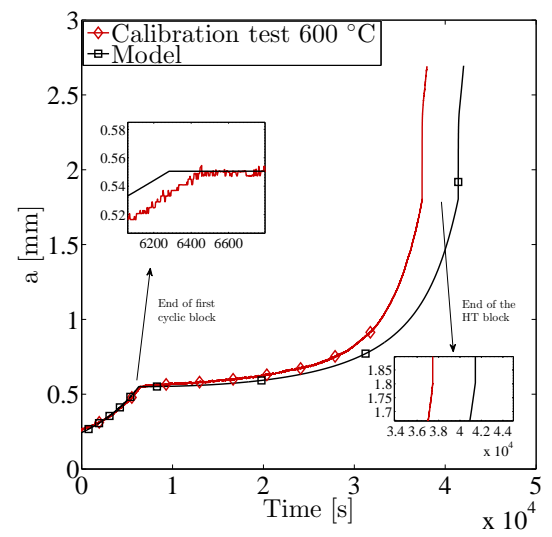

(b)

Figure 6: Calibration test for $600{ }^{\circ} \mathrm{C}$. (a) Calibration of parameters and (b) crack length vs. time. 


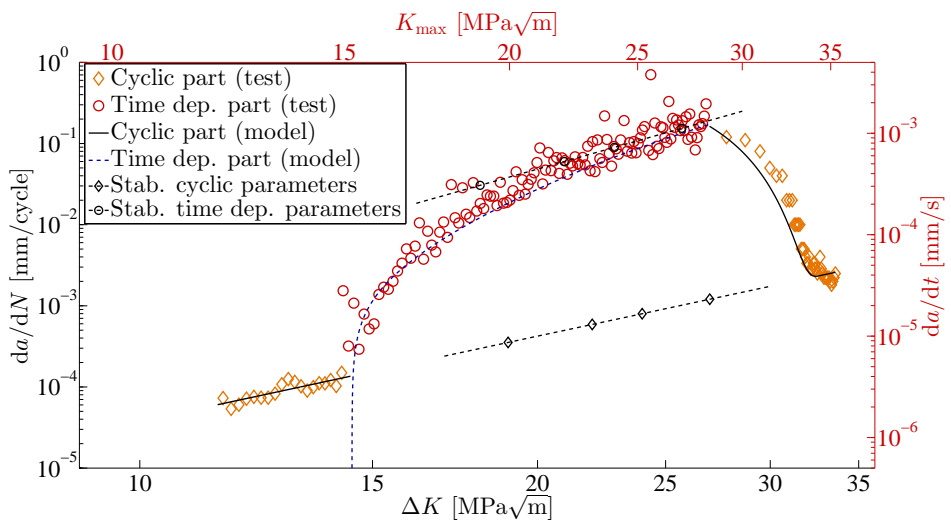

(a)

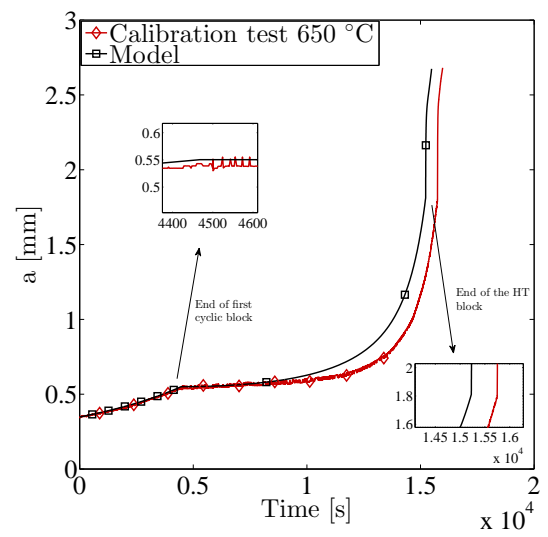

(b)

Figure 7: Calibration test for $650{ }^{\circ} \mathrm{C}$. (a) Calibration of parameters and (b) crack length vs. time.

\subsection{Validation}

In order to validate the model several different tests were performed. In total 18 tests of four different types have been studied; (A) pure time dependent tests, (B) hold time tests, (C) simple block tests and (D) more complex block tests, all seen in Fig. 8 and Table 3, where the results of all the tests, including the calibration tests can be found, and all with a final crack length of about $2.6 \mathrm{~mm}$. For more information about test types A, B and C, please see [3, 4, 23, 42]. Also note that in Table 3 , the column $t_{\exp } / t_{\text {sim. }}$. refers to the ratio between experimental time and simulated time when the crack length has reached its final value of $2.6 \mathrm{~mm}$. 

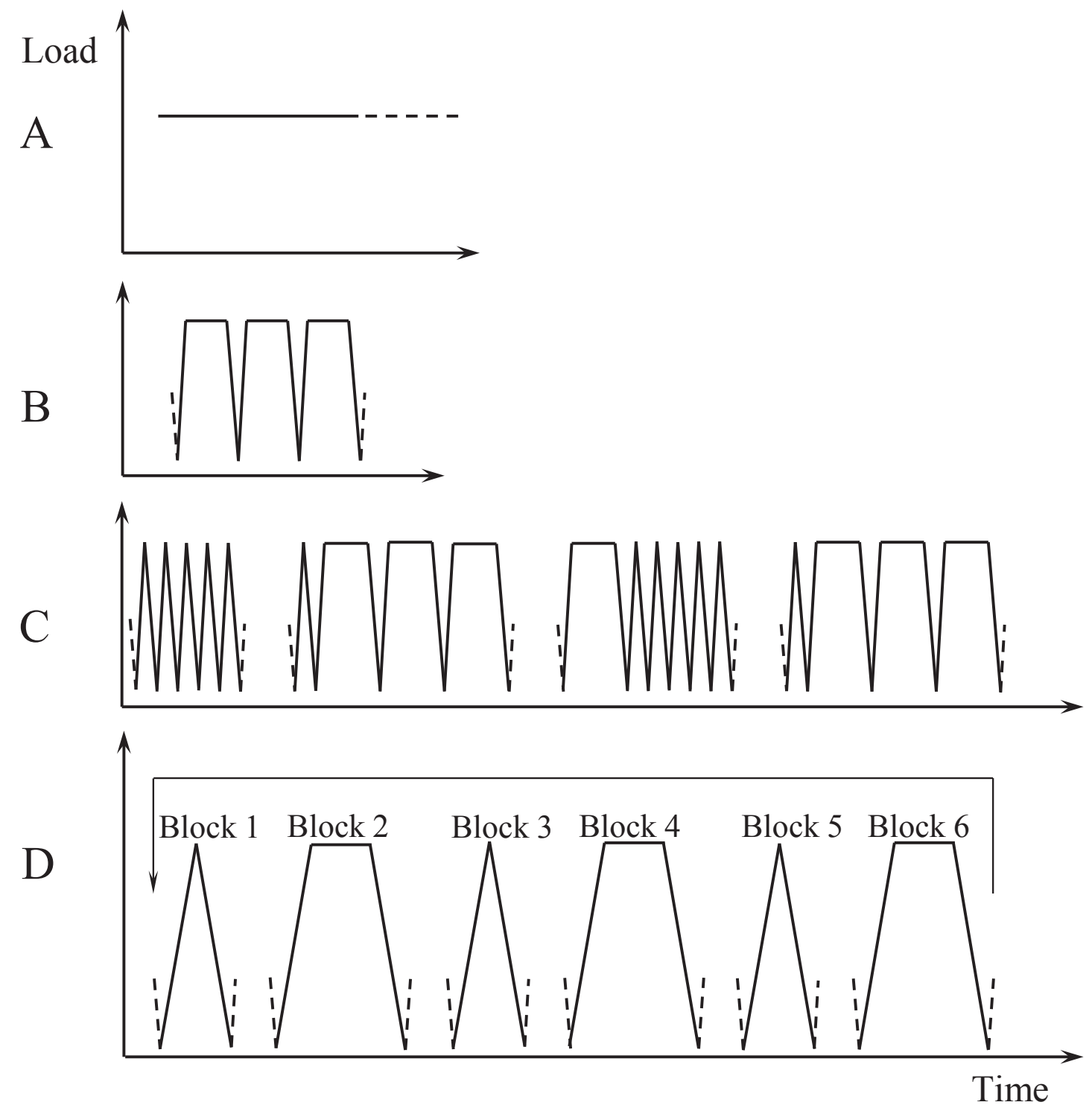

Figure 8: Validation load sequences. 
Table 3: Tests and results $\left(a_{\mathrm{final}} \approx 2.6 \mathrm{~mm}\right)$.

\begin{tabular}{|c|c|c|c|c|}
\hline Temp. $\left[{ }^{\circ} \mathrm{C}\right]$ & $\sigma_{\max }[\mathrm{MPa}]$ & Load sequence & $a_{\text {start }}[\mathrm{mm}]$ & $t_{\text {exp. }} / t_{\text {sim. }}$. \\
\hline $550^{\mathrm{a}}$ & 650 & Calibration & 0.23 & 0.96 \\
\hline $550^{\mathrm{b}}$ & 650 & $\mathrm{~A}$ & 1.32 & 1.15 \\
\hline $550^{\mathrm{b}}$ & 650 & $\mathrm{~A}$ & 0.58 & 0.89 \\
\hline $550^{\mathrm{b}}$ & 650 & B (90 s HT $)$ & 0.33 & 0.89 \\
\hline $550^{\mathrm{b}}$ & 650 & B (90 s HT $)$ & 0.47 & 1.08 \\
\hline $550^{\mathrm{b}}$ & 650 & B (2160 s HT) & 0.33 & 0.74 \\
\hline $550^{\mathrm{a}}$ & 650 & B (2160 s HT $)$ & 0.55 & 1.76 \\
\hline $550^{\mathrm{b}}$ & 650 & B (21600 s HT $)$ & 0.49 & 1.06 \\
\hline $550^{\mathrm{b}}$ & 650 & $\mathrm{C}(90 \mathrm{~s} \mathrm{HT})$ & 0.46 & 1.24 \\
\hline $550^{\mathrm{b}}$ & 650 & $\mathrm{C}(180 \mathrm{~s}$ HT $)$ & 0.41 & 1.30 \\
\hline $550^{\mathrm{b}}$ & 650 & $\mathrm{C}(2160 \mathrm{~s}$ HT $)$ & 0.45 & 0.88 \\
\hline $550^{\mathrm{b}}$ & 650 & $\mathrm{C}(21600 \mathrm{~s} \mathrm{HT})$ & 0.48 & 0.88 \\
\hline $550^{\mathrm{a}}$ & 650 & $\mathrm{D}$ & 0.52 & 1.52 \\
\hline $600^{\mathrm{b}}$ & 600 & Calibration & 0.24 & 0.90 \\
\hline $600^{\mathrm{b}}$ & 600 & $\mathrm{D}^{\mathrm{c}}$ & 0.50 & 1.05 \\
\hline $600^{\mathrm{b}}$ & 600 & $\mathrm{D}$ & 0.49 & 3.14 \\
\hline $650^{\mathrm{b}}$ & 550 & Calibration & 0.35 & 1.03 \\
\hline $650^{\mathrm{b}}$ & 510 & $\mathrm{~A}$ & 0.68 & 0.62 \\
\hline $650^{\mathrm{b}}$ & 500 & B (90 s HT) & 0.42 & 0.57 \\
\hline $650^{\mathrm{b}}$ & 550 & B (2160 s HT) & 0.73 & 2.72 \\
\hline $650^{\mathrm{b}}$ & 525 & B (2160 s HT $)$ & 0.54 & 0.61 \\
\hline $650^{\mathrm{b}}$ & 500 & $\mathrm{C}(90 \mathrm{~s}$ HT $)$ & 0.41 & 0.65 \\
\hline
\end{tabular}

The block tests of the more complex form (type D), are specified in Table 4. These tests were performed for 550 and $600{ }^{\circ} \mathrm{C}$ and the results are seen in Fig. 9 for $550{ }^{\circ} \mathrm{C}$, and in Fig. 10 for $600{ }^{\circ} \mathrm{C}$.

Table 4: Load shape for validation tests type D

\begin{tabular}{llllllll}
\hline Temp. ${ }^{\circ} \mathrm{C}$ & $\sigma_{\max }[\mathrm{MPa}]$ & Block 1 & Block 2 & Block 3 & Block 4 & Block 5 & Block 6 \\
\hline 550 & 650 & $10 \times 0.5 \mathrm{~Hz}$ & $5 \times 4000 \mathrm{~s}$ & $10 \times 0.5 \mathrm{~Hz}$ & $5 \times 8000 \mathrm{~s}$ & $10 \times 0.5 \mathrm{~Hz}$ & $3 \times 12000 \mathrm{~s}$ \\
600 & 600 & $5 \times 0.5 \mathrm{~Hz}$ & $2 \times 2000 \mathrm{~s}$ & $5 \times 0.5 \mathrm{~Hz}$ & $2 \times 4000 \mathrm{~s}$ & $5 \times 0.5 \mathrm{~Hz}$ & $1 \times 6000 \mathrm{~s}$ \\
\hline
\end{tabular}

abatch nr. 2

batch nr. 1

${ }^{\mathrm{c}}$ Test seen in Fig. 10 


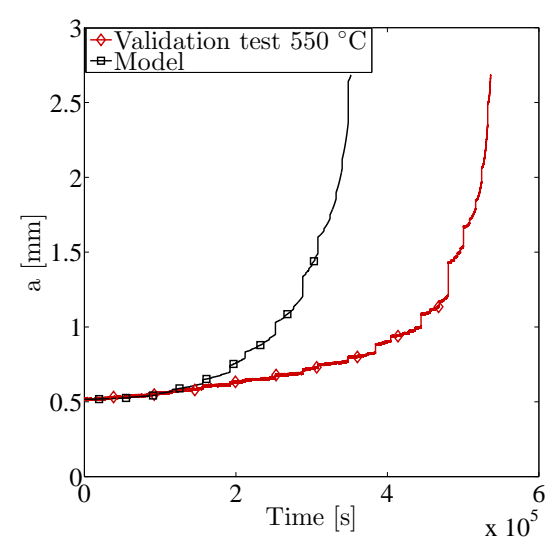

(a)

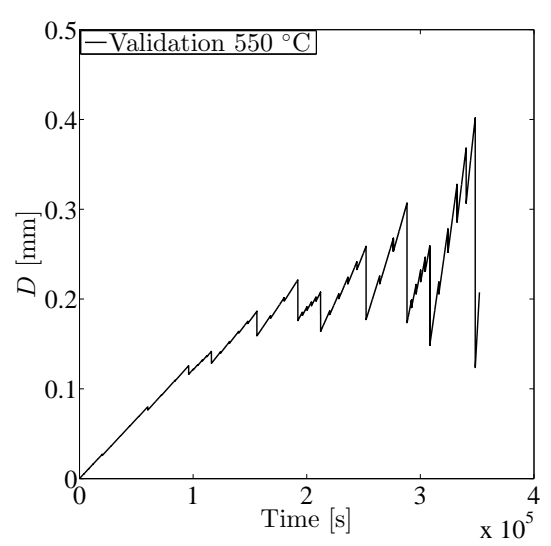

(b)

Figure 9: Validation test type D for $550{ }^{\circ} \mathrm{C}$. (a) Crack length vs. time and (b) build up and destruction of the damaged zone.

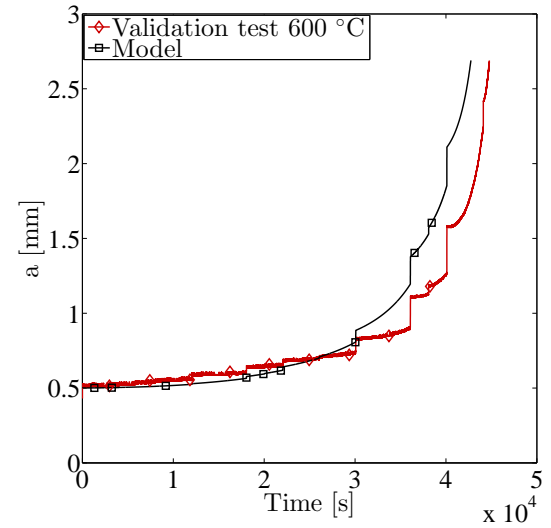

(a)

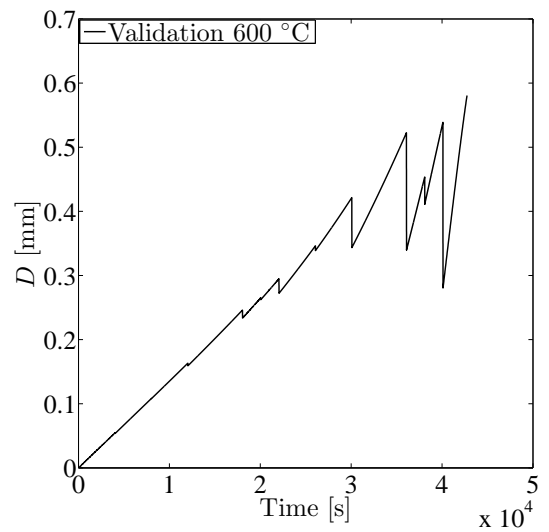

(b)

Figure 10: Validation test type D for $600^{\circ} \mathrm{C}$. (a) Crack length vs. time and (b) build up and destruction of the damaged zone.

\section{Discussion}

The history dependent phenomenological model presented in this paper is based on observations of how the embrittlement of the grain boundaries takes place. With the damaged zone concept, it is possible to describe the observed crack growth phenomena seen in the experimental testing. It should however be pointed out that the modelling paradigm does not incorporate a description of how the actual embrittlement of the grain boundaries takes place, for such works see e.g. [47].

In the previous section, simulations of 22 different tests are reported, where the calibration and validation (type D) tests have not been reported elsewhere. The calibration tests have been set up for the purpose of efficient calibration of model parameters (1 single test needed if not considering scatter), while the validation tests are meant to represent a somewhat more application relevant situation. In Fig. $9 \mathrm{~b}$ and $10 \mathrm{~b}$ the build-up and destruction of the damaged zone throughout the validation test sequence are shown, and it can be observed how the crack growth is retarded in the beginning of the next hold time. After some time the incubation 
behaviour is overcome, and as a result the crack growth rate increases, see Fig. 9a and 10a. Thus, this fundamental aspect of the crack growth behaviour seems to be realistically captured.

As seen in Table 3 the results are not consistent with the desired $t_{\text {exp. }} / t_{\text {sim. }}=1$ ratio, and the ratio is seen to deviate more as the temperature increases. However, even for tests at the same temperature and almost identical initial crack lengths a large variation can be seen, compare e.g. the two tests of type $\mathrm{D}$ at $600{ }^{\circ} \mathrm{C}$ reported in Table 3 and Fig. 11. Another example is the difference between the optimised parameters between the two different batches for $550{ }^{\circ} \mathrm{C}$ in Table 2 , where $B_{t}$, i.e. the fitting parameter which controls the incubation time, varies from 0.35 for batch nr. 2 to 0.71 for batch $\mathrm{nr}$. 1. The explanation for this might be the influence of different grain morphologies, cf. [36].

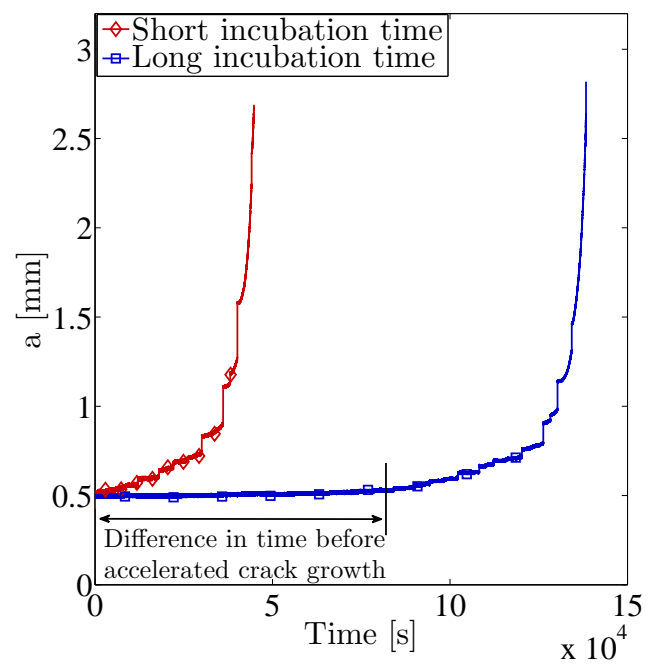

Figure 11: The two validation tests at $600{ }^{\circ} \mathrm{C}$, and their difference in time before accelerated crack growth.

In order to analyse the statistics found in Table 3, a cumulative probability plot has been set up using MATLAB Statistics Toolbox [45], see Fig. 12. As can be seen, an assumed lognormal distribution for the ratio $t_{\text {exp. }} / t_{\text {sim. }}$ seems to capture the observed behaviour, with most of the points centered around the 50th percentile. By extrapolation of the trendline to $0.1 \%$ (approximately $-3 \sigma$, where $\sigma$ stands for one standard deviation), one receives a scatter factor of 3.7 , at a probability of failure of $0.1 \%$, cf. [48], this value is of the same order as equal regular $\mathrm{d} a / \mathrm{d} N$ curves typically display [49]. However, as noted, the tests reported in Table 3 are not of the same test type, and the probability evaluation should therefore be seen as an illustration of what the model is capable of. 


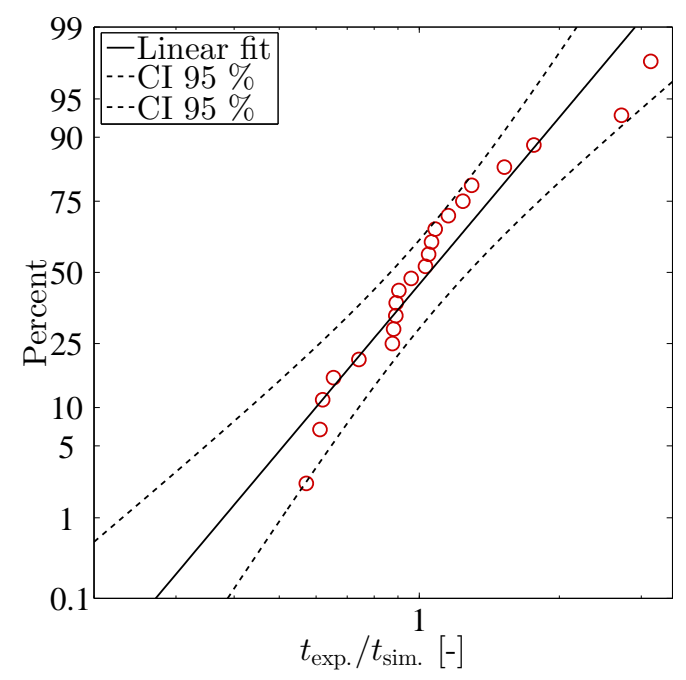

Figure 12: Log-normal distribution of $t_{\text {exp. }} / t_{\text {sim. }}=1$.

For a comparison of the model presented in this paper and the previous one in [29], where the expression for the incubation time is absent, see Fig. 13 and 14. In the previous paper [29], $C_{t}=C_{0 t}$ and $S_{t}=\left(D / D_{\max }\right)^{B_{t}}$, otherwise the model is identical with that of Section 3, with optimised values for $B_{t}$ of 1.53 for $550{ }^{\circ} \mathrm{C}$ (batch nr. 2) and 1.69 for $600{ }^{\circ} \mathrm{C}$ (batch nr. 1). As seen, the function $C_{t}$ contributes to reducing the error in time towards the experimental results.

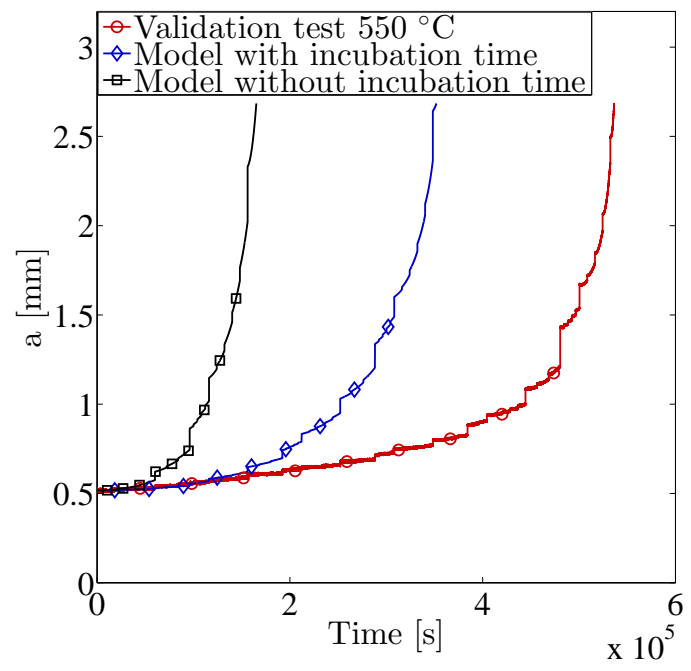

Figure 13: Comparison of the crack growth description with and without incubation time and for the validation test at $550{ }^{\circ} \mathrm{C}$ (also seen in Fig. 9a). 


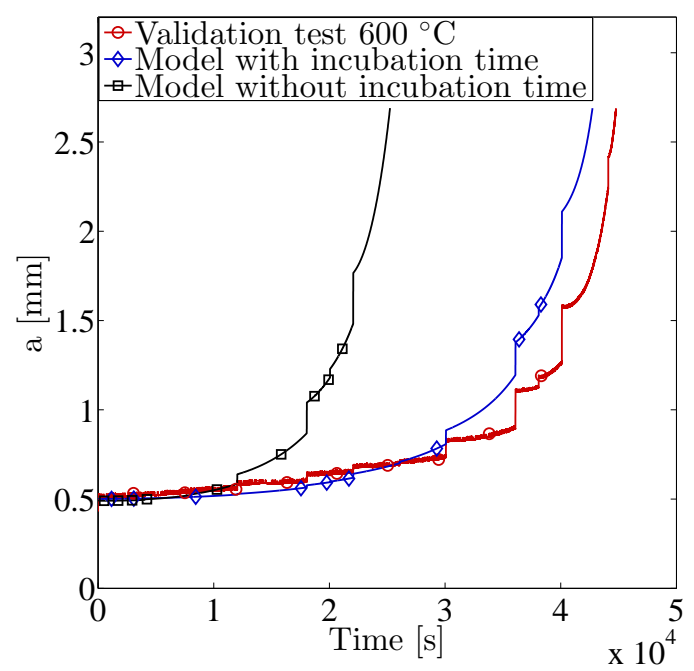

Figure 14: Comparison of the crack growth description with and without incubation time and for the validation test at $600{ }^{\circ} \mathrm{C}$ (also seen in Fig. 10a).

\section{Summary and conclusions}

Testing and simulation of the fatigue crack growth behaviour of Inconel 718 under hold time conditions have been conducted for three different temperatures, namely 550,600 and $650{ }^{\circ} \mathrm{C}$. A calibration test has been suggested for cost-efficient parameter determination. In total 22 different tests were simulated with satisfying results. The main conclusions from this are:

- The suggested calibration test shows good correlation with the model developed. However, the model seems to diverge more as temperature and damaged zone increase.

- The function $C_{t}$ developed to handle the incubation time seen in the tests, seems to be able to describe the observed behaviour, with no more fitting parameters than in [29].

- The model developed gives no larger scatter than what is typically found in regular cyclic fatigue crack propagation evaluations, with a scatter factor of 3.7 received from Fig. 12 when considering a probability of failure of $0.1 \%$.

To be able to handle more application-near conditions, the model presented must be generalised with respect to arbitrary loading rates (the regions between the previously discussed limits $X$ and $Y$, which are still to be determined), stress gradients, overloads/underloads, and different $\mathrm{R}$-values. Different $\mathrm{R}$ values are preferably handled by combining the model with a crack closure description for the cyclic part of the model (work in progress).

\section{Acknowledgements}

The authors would like to thank Mr. Patrik Nylander and Mr. Bo Skoog, Linköping University, for the laboratory work and the project teams at Linköping University, Siemens Industrial Turbomachinery AB and GKN Aerospace Engine Systems for valuable discussions. This research has been funded by the Swedish Energy Agency, Siemens Industrial Turbomachinery AB, GKN Aerospace Engine Systems, and the Royal Institute of Technology through the Swedish research programme TURBO POWER, the support of which is gratefully acknowledged.

[1] Pédron JP, Pineau A. The effect of microstructure and environment on the crack growth behaviour of Inconel 718 alloy at $650{ }^{\circ} \mathrm{C}$ under fatigue, creep and combined loading. Material Science and Engineering $1982 ; 56(2): 143-156$. 
[2] Heuler P, Affeldt E, Wanhill RJH. Effects of loading waveform and stress field on high temperature fatigue crack growth of alloy 718. Materialwissenschaft und Werkstofftechnik 2003;34(9):790-796.

[3] Gustafsson D, Moverare JJ, Johansson S, Hörnqvist M, Simonsson K, Sjöström S, Sharifimajda B. Fatigue crack growth behaviour of Inconel 718 with high temperature hold times. Procedia Engineering 2010;2(1):1095 - 1104. Fatigue 2010.

[4] Gustafsson D, Moverare JJ, Johansson S, Simonsson K, Hörnqvist M, Månsson T, Sjöström S. Influence of high temperature hold times on the fatigue crack propagation in Inconel 718. International Journal of Fatigue 2011;33(11):1461 - 1469 .

[5] Larsen JM, Nicholas T. Load sequence crack growth transients in a superalloy at elevated temperature. Fracture Mechanics: Fourteenth Symposium - Volume II: Testing and Applications, ASTM STP 791, 1983;II$536-\mathrm{II}-552$.

[6] Nicholas T, Weerasooriya T. Hold-time effects in elevated temperature fatigue crack propagation. Fracture Mechanics: Seventeenth Volume, ASTM STP 905, 1986;155 - 168.

[7] Weerasooriya T. Effect of frequency on fatigue crack growth rate of Inconel 718 at high temperature. Air Force Wright Aeronautical Laboratories Report, AFWAL-TR-87-4038, Wright-Patterson Air Force Base, OH; 1987.

[8] Andrieu E, Molins R, Ghonem H, Pineau A. Intergranular crack tip oxidation mechanism in a nickel-based superalloy. Materials Science and Engineering: A 1992;154(1):21 - 28.

[9] Ghonem H, Nicholas T, Pineau A. Elevated temperature fatigue crack growth in alloy 718-part I: Effects of mechanical variables. Fatigue \& Fracture of Engineering Materials \& Structures 1993;16(5):565-576.

[10] Ghonem H, Nicholas T, Pineau A. Elevated temperature fatigue crack growth in alloy 718-part II: Effects of environmental and material variables. Fatigue \& Fracture of Engineering Materials \& Structures 1993;16(6):577-590.

[11] Branco CM, Byrne J. Fatigue behaviour of the nickel-based superalloy IN718 at elevated temperature. Materials at High Temperatures 1994;12(4):261 - 267.

[12] Molins R, Hochstetter G, Chassaigne JC, Andrieu E. Oxidation effects on the fatigue crack growth behaviour of alloy 718 at high temperature. Acta Materialia 1997;45(2):663 - 674.

[13] Antunes FV, Ferreira JM, Branco CM, Byrne J. High temperature fatigue crack growth in Inconel 718. Materials at High Temperatures 2000;17(4):439 - 448.

[14] Antunes FV, Ferreira JM, Branco CM, Byrne J. Influence of stress state on high temperature fatigue crack growth in Inconel 718. Fatigue \& Fracture of Engineering Materials \& Structures 2001;24(2):127-135.

[15] Paris PC, Gomez MP, Anderson WE. A rotational analytic theory of fatigue. The Trend in Engineering 1961;13:9-14.

[16] Paris PC, Erdogan F. A critical analysis of crack propagation laws. Journal of Basic Engineering $1963 ; 85(4): 528-534$.

[17] Saxena A. A model for predicting the effect of frequency on fatigue crack growth behavior at elevated temperature. Fatigue \& Fracture of Engineering Materials \& Structures 1981;3(3):247-255, .

[18] Saxena A, Williams RS, Shih TT. A model for representing and predicting the influence of hold time on fatigue crack growth behavior at elevated temperature. Fracture Mechanics: Thirteenth Conferance, ASTM STP 743 1981;86 - 99 .

[19] Gayda J, Gabb TP, Miner RV. Fatigue crack propagation of nickel-base superalloys at $650{ }^{\circ} \mathrm{C}$. Low Cycle fatigue, ASTM STP 942 1988;293 - 309.

[20] Ghonem H, Zheng D. Depth of intergranular oxygen diffusion during environment-dependent fatigue crack growth in alloy 718. Materials Science and Engineering: A 1992;150(2):151 - 60.

[21] Van Stone RH, Slavik DC. Prediction of time dependent crack growth with retardation effects in nickel base alloys. fatigue and fracture mechanics: 31 st volume, ASTM STP 1389, 2000;405-426.

[22] Wackermann K, Krupp U, Christ HJ. Effects of the environment on the crack propagation behavior of IN718 in the temperature range of the dynamic embrittlement. Journal of ASTM International 2011;8(5):297-312.

[23] Gustafsson D, Moverare JJ, Simonsson K, Johansson S, Hörnqvist M, Månsson T, Sjöström S. Fatigue crack growth behaviour of Inconel 718 - the concept of a damaged zone caused by high temperature hold times. Procedia Engineering 2011;10(0):2821 - 2826. 11th International Conference on the Mechanical Behavior of Materials (ICM11).

[24] Ghonem H, Zheng D. Oxidation-assisted fatigue crack growth behavior in alloy 718-part I. quantitative modeling. Fatigue \& Fracture of Engineering Materials \& Structures 1991;14(7):749-760.

[25] Zheng D, Ghonem H. Oxidation-assisted fatigue crack growth behavior in alloy 718-part II. applications. Fatigue \& Fracture of Engineering Materials \& Structures 1991;14(7):761-768.

[26] Kruch S, Prigent P, Chaboche JL. A fracture mechanics based fatigue-creep-environment crack growth model for high temperature. International Journal of Pressure Vessels and Piping 1994;59:141-148.

[27] Gallerneau F, Kruch S, Kanouté P. A new modelling of crack propagation with fatigue-creep-oxidation interaction under non isothermal loading. Symposium on Ageing Mechanisms and Control: Part B Monitoring and Management of Gas Turbine Fleets for Extended Life and Reduced Costs, Manchester UK, 8-11 October 2001.

[28] Zhao LG, Tong J, Hardy MC. Prediction of crack growth in a nickel-based superalloy under fatigue-oxidation conditions. Engineering Fracture Mechanics 2010;77(6):925 - 38.

[29] Gustafsson D, Lundström E, Simonsson K. Modelling of high temperature fatigue crack growth in Inconel 718 under hold time conditions. International Journal of Fatigue 2013;52:124 - 130.

[30] Saxena A, Findley K. Dwell-time fatigue crack growth in Ni-base superalloys. AFRL-ML-TR-2007-4117, Air Force Research Laboratory, Wright-Patterson Air Force Base, OH; 2003. 
[31] Pineau A, Antolovich SD. High temperature fatigue of nickel-base superalloys - a review with special emphasis on deformation modes and oxidation. Engineering Failure Analysis 2009;16(8):2668 - 2697.

[32] Findley KO, Evans JL, Saxena A. A critical assessment of fatigue crack nucleation and growth models for Ni- and Ni, Fe-based superalloys. International Materials Reviews 2011;56(1):49 - 71.

[33] Bika D, Pfaendtner JA, Menyhard M, McMahon Jr CJ. Sulfur-induced dynamic embrittlement in a low-alloy steel. Acta Metallurgica et Materialia 1995;43(5):1895 - 1908.

[34] Pfaendtner JA, McMahon Jr CJ. Oxygen-induced intergranular cracking of a Ni-base alloy at elevated temperatures - an example of dynamic embrittlement. Acta Materialia 2001;49(16):3369 - 3377.

[35] Krupp U, Kane WM, Liu X, Dueber O, Laird C, McMahon Jr CJ. The effect of grain-boundary-engineeringtype processing on oxygen-induced cracking of IN718. Materials Science and Engineering: A 2003;349(1-2):213 $-217$.

[36] Krupp U, Kane WM, Laird C, McMahon CJ. Brittle intergranular fracture of a Ni-base superalloy at high temperatures by dynamic embrittlement. Materials Science and Engineering: A 2004;387-389(0):409 - 413. 13th International Conference on the Strength of Materials.

[37] Krupp U, McMahon Jr CJ. Dynamic embrittlement-time-dependent brittle fracture. Journal of Alloys and Compounds 2004;378(1-2):79 - 84. Proceedings of the 9th International Symposium on Physics of Materials (ISPMA 9).

[38] Krupp U. Dynamic embrittlement - time-dependent quasi-brittle intergranular fracture at high temperatures. International Materials Reviews 2005;50(2):83-97.

[39] Woodford DA. Gas phase embrittlement and time dependent cracking of nickel based superalloys. Energy Materials: Materials Science and Engineering for Energy Systems 2006;1(1):59-79.

[40] Liu XB, Ma LZ, Chang KM, Barbero E. Fatigue crack propagation of Ni-based superalloys. Acta Metallurgica Sinica $2005 ; 18(1): 55-64$.

[41] Diboine A, Pineau A. Creep crack initiation and growth in Inconel 718 alloy at $650{ }^{\circ} \mathrm{C}$. Fatigue \& Fracture of Engineering Materials \& Structures 1987;10(2):141-151.

[42] Gustafsson D, Lundström E. High temperature fatigue crack growth behaviour of Inconel 718 under hold time and overload conditions. International Journal of Fatigue 2013;48:178 - 186.

[43] ASTM E647-08, Standard Test Method for Measurement of Fatigue Crack Growth Rates. ASTM International, West Conshohocken, PA; 2008

[44] ASTM E740-03, Standard Practice for Fracture Testing with Surface-Crack Tension Specimens. ASTM International, West Conshohocken, PA; 2003

[45] MATLAB. Version 8.0.0.783 (R2012b). The MathWorks Inc., Natick, Massachusetts; 2012.

[46] Stander N, Roux W, Goel T, Eggleston T, Craig K. LS-OPT User's manual, Version 4.2, Livermore Software Technology Corporation, Livermore, CA; 2011.

[47] Bika D, McMahon Jr CJ. A model for dynamic embrittlement. Acta Metallurgica et Materialia 1995;43(5):1909 - 1916 .

[48] Bullen NI. A note on test factors. Reports and Memoranda No. 3166, H.M.S.O; 1956.

[49] Personal communication, GKN Aerospace Engine Systems. 